\title{
COLD DISKS: SPITZER SPECTROSCOPY OF DISKS AROUND YOUNG STARS WITH LARGE GAPS
}

\author{
J. M. Brown, ${ }^{1}$ G. A. Blake, ${ }^{2}$ C. P. Dullemond,${ }^{3}$ B. Merín, ${ }^{4}$ J. C. Augereau,${ }^{4,5}$ A. C. A. Boogert ${ }^{6}$ N. J. Evans II, \\ V. C. Geers, ${ }^{4}$ F. Lahuis, ${ }^{4,8}$ J. E. Kessler-Silacci, ${ }^{7}$ K. M. Pontoppidan, ${ }^{2}$ and E. F. van DishoecK ${ }^{4}$ \\ Received 2006 December 1; accepted 2007 June 12; published 2007 July 17
}

\begin{abstract}
We have identified four circumstellar disks with a deficit of dust emission from their inner 15-50 AU. All four stars have F-G spectral type and were uncovered as part of the Spitzer Space Telescope "Cores to Disks" Legacy Program Infrared Spectrograph (IRS) first-look survey of 100 pre-main-sequence stars. Modeling of the spectral energy distributions indicates a reduction in dust density by factors of 100-1000 from disk radii between $\sim 0.4$ and $15-50$ AU but with massive gas-rich disks at larger radii. This large contrast between the inner and outer disk has led us to use the term "cold disks" to distinguish these unusual systems. However, hot dust $\left[(0.02-0.2) M_{\text {moon }}\right]$ is still present close to the central star $(R \leq 0.8 \mathrm{AU})$. We introduce the $30 \mu \mathrm{m} / 13 \mu \mathrm{m}$ flux density ratio as a new diagnostic for identifying cold disks. The mechanisms for dust clearing over such large gaps are discussed. Although rare, cold disks are likely in transition from an optically thick to an optically thin state and so offer excellent laboratories for the study of planet formation.
\end{abstract}

Subject headings: planetary systems: protoplanetary disks — stars: pre-main-sequence

Online material: color figures

\section{INTRODUCTION}

The evolutionary processes transforming massive, gas-rich circumstellar disks into tenuous, gas-poor debris disks are still not well understood. During this crucial interval, planets form and the remaining disk material is accreted or dispersed. The path by which this transition proceeds remains uncertain. Do disks clear uniformly throughout via grain growth and settling, or is the process accelerated in the inner regions forming gaps (e.g., Alexander et al. 2006)? Evidence of dust clearing should be visible in the infrared (IR) spectral energy distribution (SED). The Spitzer Space Telescope, with its wide wavelength coverage and increased sensitivity, is starting to reveal a new population of disks with unusual SEDs.

Lack of mid-IR excess emission from disks has been interpreted as a sign of dust clearing since the first disk SEDs were observed with IRAS (Strom et al. 1989). Further examples and some spectra (e.g., HD 100546; Bouwman et al. 2003) were obtained of a few Herbig Ae/Be stars with the ISO. In this Letter, we designate disks with this characteristic missing mid-IR emission as "cold disks" due to the lack of emission from warm dust. These SEDs morphologically fall between the SEDs of classical optically thick disks, which show excess emission throughout the IR, and debris disks characterized by very weak far-IR excesses, leading them to be considered as transitional objects between the two classes.

Spitzer spectra are particularly important to characterize the sharp rise, since they cover the critical 8-30 $\mu \mathrm{m}$ range. The earliest example discovered with Spitzer was CoKu Tau 4, modeled to have an inner hole within $10 \mathrm{AU}$ (Forrest et al. 2004; D'Alessio et al. 2005). Three additional T Tauri stars, TW Hya, GM Aur,

\footnotetext{
${ }^{1}$ Division of Physics, Mathematics, and Astronomy, California Institute of Technology, Pasadena, CA 91125; jmb@astro.caltech.edu.

${ }^{2}$ Division of Geological and Planetary Sciences, California Institute of Technology, Pasadena, CA 91125.

${ }^{3}$ Max-Planck-Institut fur Astrophysik, 69117 Heidelberg, Germany.

${ }^{4}$ Leiden Observatory, Leiden University, 2300 RA Leiden, Netherlands.

${ }^{5}$ Laboratoire d'Astrophysique de l'Observatoire de Grenoble, 38041 Grenoble Cedex 9, France.

${ }^{6}$ IPAC, California Institute of Technology, Pasadena, CA 91125.

${ }^{7}$ Department of Astronomy, University of Texas, Austin, TX 78712-0259.

${ }^{8}$ SRON National Institute for Space Research, 9700 AV Groningen, Netherlands.
}

and DM Tau (Calvet et al. 2002, 2005), have been identified as also containing cleared inner regions. One of the most exciting proposed explanations for these cold disks is that a planet has cleared a gap in the disk and thus that these inner holes may trace the presence of planetary systems (Varnière et al. 2006).

In this Letter, we present Spitzer spectra of four young stars of $\mathrm{F}-\mathrm{G}$ spectral type that show a deficit of dust emission from the inner 15-50 AU of the disk yet strong excesses longward of $20 \mu \mathrm{m}$. These cold disk sources were identified in the firstlook survey of the Cores to Disks (c2d) Spitzer Legacy Project (Evans et al. 2003), comprising $~ 100$ young (<10 Myr) stars, mainly T Tauri K and M stars, with circumstellar disks. All four sources are characterized by a steep, $\sim 10 \times$ rise in flux beginning at $15 \mu \mathrm{m}$, indicating a sudden change in disk properties at a specific radius in a manner similar to that seen in the $\mathrm{T}$ Tauri transitional disks. Such objects are rare but provide an important window into disk evolution and planet formation processes.

\section{OBSERVATIONS}

The 5-35 $\mu \mathrm{m}$ spectra were taken with the Infrared Spectrometer (IRS; Houck et al. 2004) on Spitzer using both low-resolution $\left(R=160, \lambda_{\mathrm{SL}}: 5.2-14.5 \mu \mathrm{m}, \lambda_{\mathrm{LL}}: 14.0-38.0 \mu \mathrm{m}\right)$ and high-resolution $\left(R=600, \lambda_{\mathrm{SH}}: 9.9-19.6 \mu \mathrm{m}, \lambda_{\mathrm{LH}}: 18.7-37.2 \mu \mathrm{m}\right)$ modules. Spectra were extracted from the Spitzer Science Center (SSC) Basic Calibrated Data (BCD) images, generated by pipeline S13. For the low-resolution spectra, the SSC pipeline fullaperture extraction was used. For the high-resolution modules, the $\mathrm{c} 2 \mathrm{~d}$ extraction, based on a combined sinc fitting of the spectral trace to account for bad pixels and background emission, was used (see Lahuis et al. 2006 for further details).

MIPS SED spectra, taken as part of the c2d project, are included in the SEDs for all four cold disk sources. For each source, the BCD images were co-added using MOPEX. The co-added images were extracted with IRAF, using an optimized extraction with a three- or five-column wide aperture.

\section{DISTINGUISHING CHARACTERISTICS}

The large $(\sim 100$ object) c2d IRS first-look sample allows comparison between the four cold disk sources and the remaining systems to identify trends that might be diagnostic of 


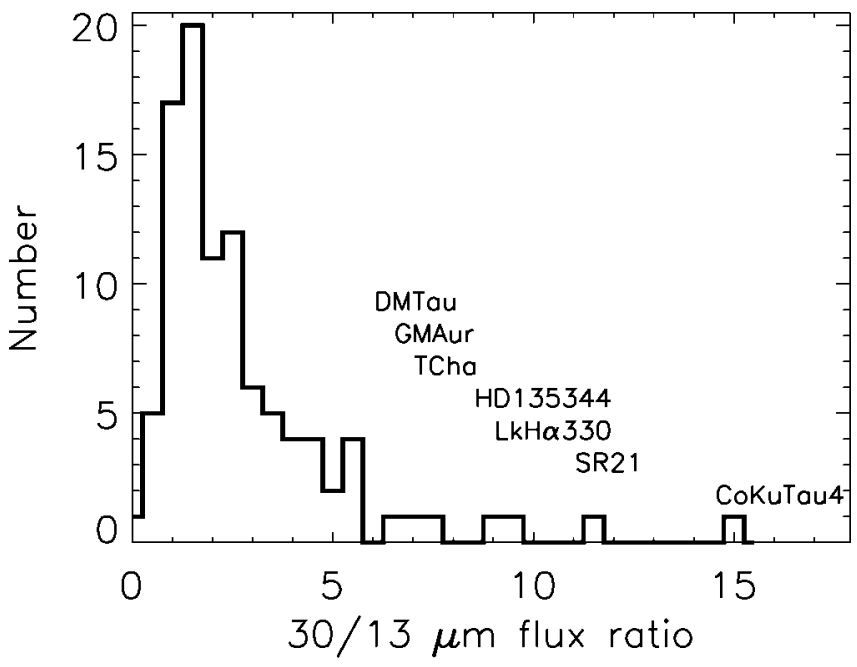

FIG. 1.-Spitzer IRS $30 \mu \mathrm{m} / 13 \mu \mathrm{m}$ flux ratios for the c2d first-look disk sample, along with those of DM Tau, GM Aur, and CoKu Tau 4. The cold disks have much larger $30 \mu \mathrm{m} / 13 \mu \mathrm{m}$ ratios than does the majority of the sample. Outliers are labeled.

their evolutionary state. The small number of cold disk sources ( $<5 \%$ identified), both in the c2d sample and in the literature, suggests that this condition is rare either due to rapid evolutionary timescales undergone by all stars or due to an unusual condition unique to a small sample of stars.

The four cold disk sources are clearly differentiated from the majority of the c2d star+disk systems by the $\sim 10$-fold increase in IR flux between 10 and $30 \mu \mathrm{m}$. To characterize this increase in dust emission, we have used the $30 \mu \mathrm{m} / 13 \mu \mathrm{m}$ flux ratio (see Fig. 1). These wavelengths have been chosen to avoid strong silicate features but include the full increase in continuum emission. The majority of the sample has emission that increases by a factor of $2.3 \pm 1.4$ between 13 and $30 \mu \mathrm{m}$ while the transitional disks rise by factors of 5-15.

Interestingly, out of a sample comprised of predominantly lowmass stars, the cold disk sources are all of intermediate mass with spectral types of $\mathrm{F}$ and $\mathrm{G}$. The majority of $\mathrm{K}$ and $\mathrm{M}$ stars and the higher mass A and B stars have low $30 \mu \mathrm{m} / 13 \mu \mathrm{m}$ ratios. However, there are only a handful of A and B stars in our sample, so it is difficult to draw any significant conclusions about such stars.

All four cold disk sources show polycyclic aromatic hydrocarbon (PAH) features, particularly at $11.3 \mu \mathrm{m}$ (see Fig. 2). Such emission is uncommon in the c2d sample, with only $~ 10 \%$ displaying PAH features (Geers et al. 2006; see their Fig. 5 for blowups of the PAH bands). The presence of PAH features may be enhanced in these cold disks because the lack of dust in the inner disk lowers the mid-IR continuum flux creating a stronger line-to-continuum ratio, thus facilitating detection.

The four disks are also characterized by weak to nonexistent $10 \mu \mathrm{m}$ amorphous silicate features. $\mathrm{LkH} \alpha 330$ is the only one that shows an unambiguous, although low-contrast, $10 \mu \mathrm{m}$ feature. The spectrum of HD 135344 includes the wavelength region of the full $10 \mu \mathrm{m}$ band but shows no silicate feature. The spectra of SR 21 and T Cha begin at $10 \mu \mathrm{m}$ but appear to have only weak silicate emission, if any. The broader $20 \mu \mathrm{m}$ silicate feature is harder to isolate from continuum dust emission, particularly with the sharp rise in the SED beyond $15 \mu \mathrm{m}$. However, some $20 \mu \mathrm{m}$ emission does seem to be present. This feature traces colder regions of the disk than does the $10 \mu \mathrm{m}$ feature and thus indicates the presence of amorphous silicates farther out in the disks.

The $\mathrm{H} \alpha$ equivalent width is often used as a tracer of accretion.

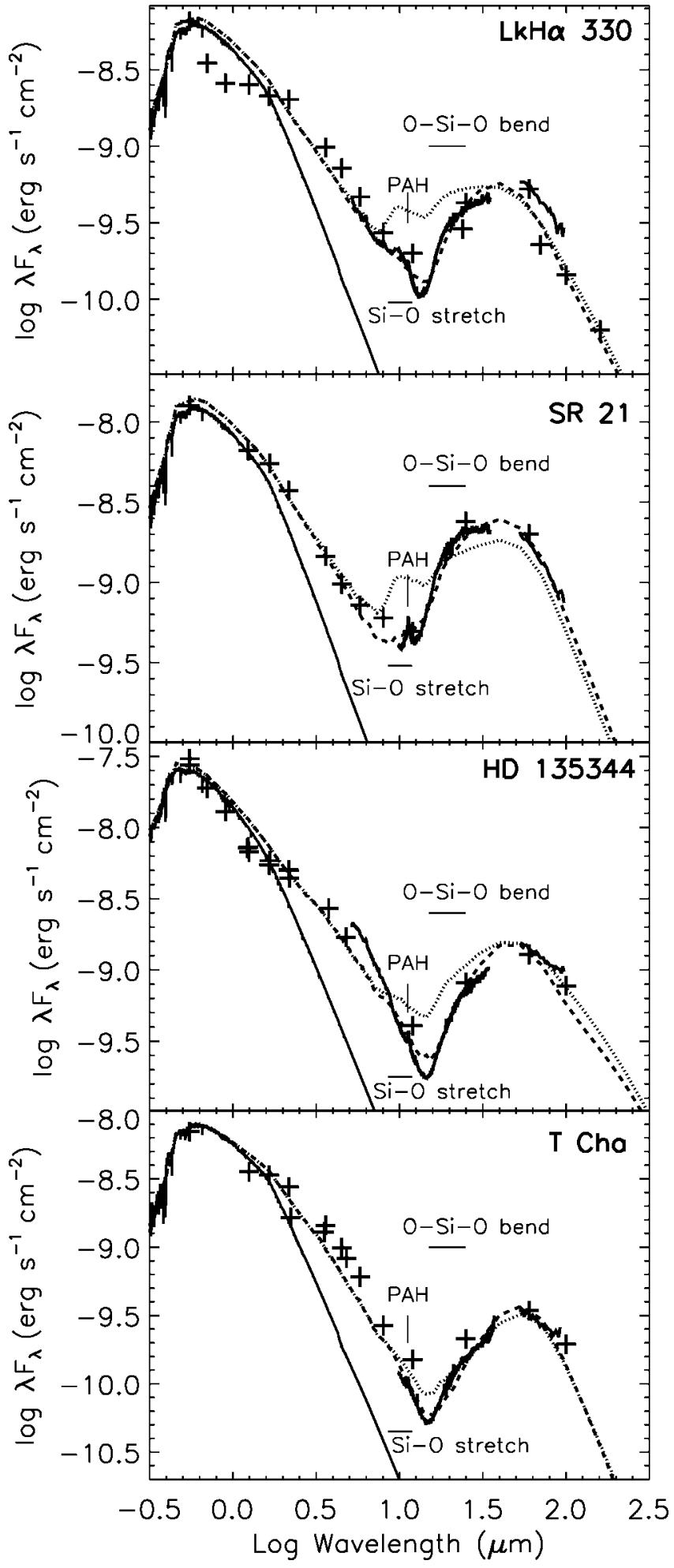

FIG. 2.-Spitzer IRS and MIPS spectra of the four cold disk sources reported here. The dashed line is the best-fit star+disk model with a gap (see Table 1), and the dotted line is the equivalent model with no gap. $J H K$ photometry is from 2MASS. IRAS photometry and our Spitzer photometry is shown in the mid- to far-IR. Optical and submillimeter photometry is from the literature (Alcalá et al. 1993; André \& Montmerle 1994; Fernandez et al. 1995; Henning et al. 1993; Osterloh \& Beckwith 1995). [See the electronic edition of the Journal for a color version of this figure.] 
TABLE 1

Model Parameters

\begin{tabular}{|c|c|c|c|c|c|c|c|c|c|c|c|c|}
\hline Source & $\begin{array}{c}\text { Spectral } \\
\text { Type }\end{array}$ & $\begin{array}{c}A_{v} \\
\text { (mag) }\end{array}$ & $\begin{array}{l}\text { Distance } \\
(\mathrm{pc})\end{array}$ & $\begin{array}{c}M_{*} \\
\left(M_{\odot}\right)\end{array}$ & $\begin{array}{l}T_{\text {eff }} \\
(\mathrm{K})\end{array}$ & $\begin{array}{c}L_{*} \\
\left(L_{\odot}\right) \\
\end{array}$ & $\begin{array}{l}R_{\text {disk, in }} \\
(\mathrm{AU})\end{array}$ & $\begin{array}{l}R_{\text {gap, in }} \\
\text { (AU) }\end{array}$ & $\begin{array}{c}R_{\text {gap, out }} \\
(\mathrm{AU})\end{array}$ & $\begin{array}{c}M_{\text {dust, small }} \\
\left(10^{-6} M_{\odot}\right) \\
\end{array}$ & $\begin{array}{c}M_{\text {inner }} \\
\left(M_{\text {lunar }}\right) \\
\end{array}$ & $H_{p}\left(R_{\text {disk }}\right) / R_{\text {disk }}$ \\
\hline $\mathrm{LkH} \alpha 330$ & $\mathrm{G}^{\mathrm{a}}$ & $1.8^{\mathrm{a}}$ & $250^{\mathrm{b}}$ & $2.5^{\mathrm{c}}$ & 5800 & 16 & 0.27 & 0.8 & 50 & 5 & 0.24 & 0.15 \\
\hline SR 21 & $\mathrm{G} 2.5^{\mathrm{d}}$ & $9^{\mathrm{d}}$ & 160 & $2.5^{\mathrm{d}}$ & 5800 & 24 & 0.25 & 0.45 & 18 & 15 & 0.10 & 0.17 \\
\hline HD 135344 & $\mathrm{~F} 4^{\mathrm{e}}$ & $0.5^{\mathrm{f}}$ & $84^{e}$ & 1.8 & $6600^{\mathrm{e}}$ & 6.8 & 0.18 & 0.45 & 45 & 5 & 0.10 & 0.13 \\
\hline T Cha ...... & $G 8^{\mathrm{g}}$ & $1.5^{\mathrm{g}}$ & $66^{\mathrm{h}}$ & $1.5^{\mathrm{g}}$ & 5600 & 1.4 & 0.08 & 0.2 & 15 & 3 & 0.025 & 0.11 \\
\hline
\end{tabular}

ReferenCES. - (a) Cohen \& Kuhi 1979; (b) Enoch et al. 2006; (c) Osterloh \& Beckwith 1995; (d) Prato et al. 2003; (e) Dunkin et al. 1997;

(f) Malfait et al. 1998; (g) Alcalá et al. 1993; (h) Wichmann et al. 1998.

Different dividing lines between nonaccreting weak-line $\mathrm{T}$ Tauri (WTT) stars and classical T Tauri (CTT) stars have been proposed, but it makes little difference to the classifications in these cases. SR $21(0.54 \AA$ in absorption: Martin et al. 1998) and T Cha (210 Å: Gregorio-Hetem et al. 1992; Alcala et al. 1995) are nominally WTT stars, although T Cha is highly variable and close to the cutoff. LkH $\alpha 330$ (11-20 Å: Fernandez et al. 1995; Cohen \& Kuhi 1979) and HD 135344 (17.4 $\AA$ : Acke et al. 2005) are clearly CTT stars.

\section{MODELING}

Modeling is necessary in order to interpret the SEDs in terms of the physical structure of the disk. In particular, can a disk model with a gap accurately reproduce the SEDs, including the steep rise between 13 and $30 \mu \mathrm{m}$ ? Is such a fit possible without resorting to a gap?

The disks were modeled with the two-dimensional radiative transfer code RADMC (Dullemond \& Dominik 2004). The steep factor of 10 rise in flux between 13 and $30 \mu \mathrm{m}$ prevents these disks from being fit well by conventional disk models. We introduce a very wide gap with an inner radius, $R_{\text {gap in }}$, near $1 \mathrm{AU}$ and an outer radius, $R_{\text {gap, out }}$, near $30 \mathrm{AU}$ (see Table 1 for specific values). In order to model the steep change in emission associated with $R_{\text {gap, out }}$, grid refinement is introduced at $R_{\text {gap, out }}$ as well as the inner dust rim, $R_{\text {disk, in }}$. A physical reduction in dust density is only one possible scenario that could result in this SED shape (see $\S 5$ ).

Input parameters for these models include the stellar mass $M_{*}$, radius $R_{*}$, and effective temperature $T_{\text {eff }}$. Kurucz models are used for the stellar photospheres. Where possible, values for stellar and disk properties are taken from the literature (see Table 1). The
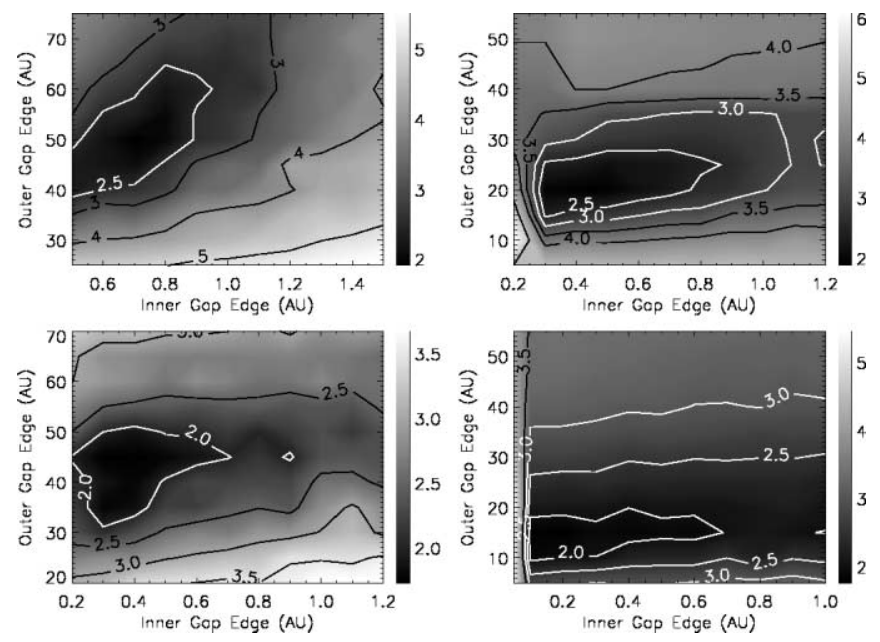

FIG. 3.-The $\chi^{2}$ contour maps of two-dimensional disk model fits to the SEDs with different inner and outer gap radii (clockwise from top left: $\mathrm{LkH} \alpha$ 330, SR 21, T Cha, and HD 135344). [See the electronic edition of the Journal for a color version of this figure.]
Siess et al. (2000) pre-main-sequence stellar tracks were used to check that the parameters were consistent. The effects of modest differences in the stellar properties on the mid-IR portions of the spectra are small. For example, a $100 \mathrm{~K}$ change in the stellar temperature results in a change in $R_{\text {gap out }}$ of $2-3 \mathrm{AU}$. Optical and (sub)millimeter photometry is often not simultaneous and is dereddened using the extinction law of Draine (2003).

The disks are assumed to be flared with surface height $H$ such that $H / R \propto R^{2 / 7}$ as in Chiang \& Goldreich (1997). The models do not calculate the hydrostatic equilibrium self-consistently, and here the pressure scale height is set at the outer disk edge, $R_{\text {disk }}$, which is assumed to be $300 \mathrm{AU}$ for all the models. Values of $H / R_{\text {disk }}$ slightly lower than predicted by hydrostatic equilibrium are needed to fit the outer disks of both HD 135344 and T Cha, which produces a smaller silicate feature even in the models with no gap. This could indicate dust settling in the outer disk, which might be expected from the older ages (8-10 vs. 2-3 Myr) inferred from the Siess et al. (2000) tracks. The dust composition is set in all models to a silicate : carbon ratio of $4: 1$. The grain sizes range from 0.01 to $10 \mu \mathrm{m}$ with a power-law index of -3.5 and a dust mass of $M_{\text {dust, small }}$. The upper size limit of $10 \mu \mathrm{m}$ is large compared to interstellar grains (Mathis et al. 1977) but was necessary to account for the lack of any $10 \mu \mathrm{m}$ amorphous silicate features. This indicates a skewing of the size distribution likely through grain growth. No crystalline silicates are included. A midplane layer of $2 \mathrm{~mm}$ grains containing $90 \%$ of the total dust mass, with the remaining $10 \%$ in $M_{\text {dust, small }}$, has been added to account for the (sub)millimeter slope (as in Dullemond \& Dominik 2004). The inner edge of the disk, $R_{\text {disk, in }}$, was set at the radius where the dust sublimation temperature of $T_{\text {dust }} \sim 1500 \mathrm{~K}$ is reached. A slightly puffed up inner rim was included with $H / R_{\text {disk, in }}$ being set at 0.03 , just above the hydrostatic equilibrium value. The disk inclinations are not well constrained, so $i \sim 30^{\circ}$ was used arbitrarily for SR 21 and $\mathrm{LkH} \alpha 330$. T Cha is more edge-on, so $i \sim 75^{\circ}$ was adopted (Alcalá et al. 1993), and $\mathrm{HD}$ 135344 is more face-on with $i \sim 10^{\circ}$ (Dent et al. 2005). However, until the disk is nearly edge-on and begins to obscure the central star, varying the inclination produces little effect on the gap properties.

The optimal size of the gap necessary to fit the mid-IR is found by minimizing the $\chi^{2}$ of the fit (see Fig. 3); $R_{\text {gap, out }}$ is tightly constrained by the steep rise at $13 \mu \mathrm{m}$, while $R_{\text {gap , in }}$ is less well constrained. The dust density in the gap was reduced by $10^{-6}$ compared to the equivalent models with no gaps, and the minimum reductions needed were factors of 10-100.

All of our sources have 1-10 $\mu \mathrm{m}$ excesses that require (0.02-0.24) $M_{\text {moon }}$ of dust between $R_{\text {disk, in }}$ and $R_{\text {gap, in }}$, a fraction of only $10^{-7}$ to $10^{-6}$ of the total refractory dust mass available. This matter is optically thick to the stellar light at the midplane. If we remove all matter from the disk inward of $R_{\text {gap, out }}$, then the near-IR fluxes are underpredicted. These excesses clearly show that at least some dust must be close to the star. We model the 1-10 $\mu \mathrm{m}$ excess by keeping the inner disk intact and only 
inserting a gap from $R_{\text {gap, in }}$ to $R_{\text {gap, out }}$, where $R_{\text {gap, in }}>R_{\text {disk, in. For }}$ $\mathrm{T}$ Cha, this results in a particularly small, hot dust region inside the gap that would have a very short lifetime, of order thousands of years at most, without a continual influx of material.

\section{DISCUSSION}

We have identified four cold disks around $F$ and $G$ type stars with unusually steep flux rises between 10 and $30 \mu \mathrm{m}$, whose SEDs can only be modeled with wide gaps of inner radii of 0.2-0.8 AU and outer radii $15-50 \mathrm{AU}$. These gaps are generally larger than those inferred for the four T Tauri stars with similar $30 \mu \mathrm{m} / 13 \mu \mathrm{m}$ ratios found so far, which have outer gap radii of 10-24 AU. Another difference is that our sources have small $1-10 \mu \mathrm{m}$ excesses, which demand that hot dust exists between the inner edge of the gap and the star; i.e., our sources have gaps rather than holes. The statistics on sources of different spectral types are still too small to conclude whether this is a general trend or peculiar to our sources and whether this extends to Herbig Ae/Be stars.

Dust clearing related to planet formation would be one of the most exciting explanations for the observed SEDs. There is also the possibility of the disk being disrupted and the dust cleared by a stellar companion. HD 135344 and SR 21 are part of wide binaries with separations of $20.4^{\prime \prime}$ and 6.4", respectively (Coulson \& Walther 1995; Prato et al. 2003). Although no companions are currently known within the modeled $R_{\text {disk }}$, close binaries $(<50 \mathrm{AU})$ cannot be ruled out for any of the sources.

Another proposed scenario for quickly clearing the inner disk region is photoevaporation (Clarke et al. 2001; Alexander et al. 2006). This physical process occurs when the photoevaporation rate driven by the ionizing flux from the central star matches the viscous accretion rate, resulting in an inner hole. The predicted size of the inner hole is given by $R_{g}=G M_{*} / c_{s}^{2}$, with $T \sim 10^{4} \mathrm{~K}$ to give $c_{s} \sim 10 \mathrm{~km} \mathrm{~s}^{-1}$, and leads to predicted hole radii of 13-18 AU for 1.5-2 $M_{\odot}$ stars, although a more rigorous examination of the gas dynamics revealed that this equation overestimates the inner hole size (Liffman 2003). However, models of Herbig Ae stars $\left(M_{*}=\right.$ $2.5 M_{\odot}$ ) that combine photoevaporation with viscous evolution and differential radial motions of dust and gas predict that the inner disk clears quickly but leaves gas-poor dust rings at 10-100 AU (Takeuchi et al. 2005). The accretion rates for all four cold disks, with the possible exception of SR 21, are too high to make this scenario likely.

An alternative to physically removing the dust is to let it grow beyond the size at which it efficiently radiates as a blackbody so that it no longer emits strongly in the mid-IR (Tanaka et al. 2005). There is general evidence for grain growth in disks from both mid-IR and millimeter data (Kessler-Silacci et al. 2006; Rodmann et al. 2006). The lack of strong $10 \mu \mathrm{m}$ amorphous silicate features also points to grains having grown beyond interstellar sizes. There has been much recent modeling work on clearing disks through grain growth. Dullemond \& Dominik (2005) found that cold disk SEDs could be produced by dust coagulation, but the timescales were too fast. Replenishment of the dust was necessary to match observed lifetimes of the disks. If replenishment processes such as fragmentation occur preferentially in the innermost region due to higher temperatures and densities (Kenyon \& Bromley 2004), this might be sufficient to produce the fraction of a lunar mass needed close to the star. Rice et al. (2006) invoke dust filtration by an embedded planet whereby large grains pile up at the outer edge of the gap while small grains and gas pass through, thus accounting for both the hot small dust grains needed to fit the near-IR and the high mass accretion rates. The high spatial resolution of the Atacama Large Millimeter/submillimeter Array should be able to test these scenarios by searching for and imaging the emission from (sub)millimeter to centimeter sized grains.

Support for this work, part of the Spitzer Legacy Science Program, was provided by NASA through contracts 1224608,1230779 , and 1256316 issued by the Jet Propulsion Laboratory, California Institute of Technology, under NASA contract 1407. Astrochemistry in Leiden is supported by a NWO Spinoza grant and a NOVA grant, and by the EU RTN-PLANETS (HPRN-CT-2002-00308). B. M. acknowledges the financial support from the Fundacion Ramon Areces (Spain). We thank the Lorentz Center in Leiden for hosting several meetings that contributed to this Letter.

\section{REFERENCES}

Acke, B., van den Ancker, M. E., \& Dullemond, C. P. 2005, A\&A, 436, 209

Alcalá, J. M., Covino, E., Franchini, M., Krautter, J., Terranegra, L., \& Wichmann, R. 1993, A\&A, 272, 225

Alcalá, J. M., Krautter, J., Schmitt, J. H. M. M., Covino, E., Wichmann, R., \& Mundt, R. 1995, A\&AS, 114, 109

Alexander, R. D., Clarke, C. J., \& Pringle, J. E. 2006, MNRAS, 369, 229

André, P., \& Montmerle, T. 1994, ApJ, 420, 837

Bouwman, J., de Koter, A., Dominik, C., \& Waters, L. B. F. M. 2003, A\&A, 401, 577

Calvet, N., D'Alessio, P., Hartmann, L., Wilner, D., Walsh, A., \& Sitko, M. 2002, ApJ, 568, 1008

Calvet, N., et al. 2005, ApJ, 630, L185

Chiang, E. I., \& Goldreich, P. 1997, ApJ, 490, 368

Clarke, C. J., Gendrin, A., \& Sotomayor, M. 2001, MNRAS, 328, 485

Cohen, M., \& Kuhi, L. V. 1979, ApJS, 41, 743

Coulson, I. M., \& Walther, D. M. 1995, MNRAS, 274, 977

D'Alessio, P., et al. 2005, ApJ, 621, 461

Dent, W. R. F., Greaves, J. S., \& Coulson, I. M. 2005, MNRAS, 359, 663

Draine, B. T. 2003, ApJ, 598, 1017

Dullemond, C. P., \& Dominik, C. 2004, A\&A, 417, 159

Dullemond, C. P., \& Dominik, C. 2005, A\&A, 434, 971

Dunkin, S. K., Barlow, M. J., \& Ryan, S. G. 1997, MNRAS, 286, 604

Enoch, M. L., et al. 2006, ApJ, 638, 293

Evans, N. J., et al. 2003, PASP, 115, 965

Fernandez, M., Ortiz, E., Eiroa, C., \& Miranda, L. F. 1995, A\&AS, 114, 439

Forrest, W. J., et al. 2004, ApJS, 154, 443

Geers, V. C., et al. 2006, A\&A, 459, 545
Gregorio-Hetem, J., Lepine, J. R. D., Quast, G. R., Torres, C. A. O., \& de La Reza, R. 1992, AJ, 103, 549

Henning, T., Pfau, W., Zinnecker, H., \& Prusti, T. 1993, A\&A, 276, 129

Houck, J. R., et al. 2004, ApJS, 154, 18

Kenyon, S. J., \& Bromley, B. C. 2004, AJ, 127, 513

Kessler-Silacci, J., et al. 2006, ApJ, 639, 275

Lahuis, F., et al. 2006, Data Legacy of the "Cores to Disks" Spectroscopy Program (Pasadena: Caltech), http://ssc.spitzer.caltech.edu/legacy/c2dhistory.html

Liffman, K. 2003, Publ. Astron. Soc. Australia, 20, 337

Malfait, K., Bogaert, E., \& Waelkens, C. 1998, A\&A, 331, 211

Martin, E. L., Montmerle, T., Gregorio-Hetem, J., \& Casanova, S. 1998, MNRAS, 300, 733

Mathis, J. S., Rumpl, W., \& Nordsieck, K. H. 1977, ApJ, 217, 425

Osterloh, M., \& Beckwith, S. V. W. 1995, ApJ, 439, 288

Prato, L., Greene, T. P., \& Simon, M. 2003, ApJ, 584, 853

Rice, W. K. M., Armitage, P. J., Wood, K., \& Lodato, G. 2006, MNRAS, 373, 1619

Rodmann, J., Henning, T., Chandler, C. J., Mundy, L. G., \& Wilner, D. J. 2006, A\&A, 446, 211

Siess, L., Dufour, E., \& Forestini, M. 2000, A\&A, 358, 593

Strom, K. M., Strom, S. E., Edwards, S., Cabrit, S., \& Skrutskie, M. F. 1989, AJ, 97, 1451

Takeuchi, T., Clarke, C. J., \& Lin, D. N. C. 2005, ApJ, 627, 286

Tanaka, H., Himeno, Y., \& Ida, S. 2005, ApJ, 625, 414

Varnière, P., Blackman, E. G., Frank, A., \& Quillen, A. C. 2006, ApJ, 640, 1110

Wichmann, R., Bastian, U., Krautter, J., Jankovics, I., \& Rucinski, S. M. 1998, MNRAS, 301, L39 